\title{
Hankel type operators on the unit disk
}

\author{
by \\ Jie Miao (State University, AR)
}

\begin{abstract}
We study Hankel operators and commutators that are associated with a symbol and a kernel function. If the kernel function satisfies an upper bound condition, we obtain a sufficient condition for commutators to be bounded or compact. If the kernel function satisfies a local bound condition, the sufficient condition turns out to be necessary. The analytic and harmonic Bergman kernels satisfy both conditions, therefore a recent result by $\mathrm{Wu}$ on Hankel operators on harmonic Bergman spaces is extended.
\end{abstract}

1. Introduction. Let $D$ denote the open unit disk in the complex plane $\mathbb{C}$ and $A$ denote the normalized Lebesgue area measure on $D$. For $p \geq 1$, let $L^{p}$ denote $L^{p}(D, d A)$. Let $P$ denote an integral operator with a kernel function $K$ on $D \times D$ given by

$$
P(g)(z)=\int_{D} g(w) K(z, w) d A(w) .
$$

For $f \in L^{1}$, we define the Hankel operator $H_{f}=(I-P) M_{f} P$ and the commutator $C_{f}=M_{f} P-P M_{f}$, where $M_{f}$ is the multiplication operator defined by $M_{f}(g)=f g$. The function $f$ is called the symbol of $H_{f}$ and $C_{f}$. There is a close relation between $H_{f}$ and $C_{f}$ when $P^{2}=P$. In this paper, we study the boundedness and compactness of $C_{f}$ that is associated with a kernel function $K(z, w)$ on $L^{p}$.

Let $L_{\mathrm{a}}^{2}=\left\{f \in L^{2}: f\right.$ is analytic on $\left.D\right\}$ denote the analytic Bergman space and let $P$ denote the orthogonal projection from $L^{2}$ onto its closed subspace $L_{\mathrm{a}}^{2}$. Then the corresponding Hankel operators and commutators have been well studied; see [1], [4], and [7]. In this case $P$ is an integral operator with the kernel function

$$
K_{\mathrm{a}}(z, w)=\frac{1}{(1-z \bar{w})^{2}} .
$$

Another case that was recently studied in [8] is the Hankel operators on the harmonic Bergman space $L_{\mathrm{h}}^{2}=\left\{f \in L^{2}: f\right.$ is harmonic on $\left.D\right\}$. The

2000 Mathematics Subject Classification: 47B35, 46E22. 
orthogonal projection $P$ from $L^{2}$ onto its closed subspace $L_{\mathrm{h}}^{2}$ is an integral operator with the kernel function (see page 357 of [9] on how it can be derived)

$$
K_{\mathrm{h}}(z, w)=2 \Re \frac{1}{(1-z \bar{w})^{2}}-1 .
$$

$\mathrm{Wu}[8]$ obtained characterizations for the boundedness and compactness of $H_{f}$ and $C_{f}$ when $f$ is a harmonic function on $D$.

The study of Hankel operators on the analytic and harmonic Bergman spaces often depends on specific techniques that are only effective for analytic or harmonic functions. As we will show, there is a necessary and sufficient condition for $C_{f}$ to be bounded or compact if the kernel function satisfies an upper bound condition and a local bound condition. Descriptions for both conditions on the kernel can be stated easily and do not rely on analytic or harmonic functions. The proof of the sufficiency is essentially the same as in [4]. The technique using a local estimation for the kernel to prove the necessity is new. We use the unit disk as our setting in this paper since it is easier to present concrete examples of kernel functions that satisfy both conditions.

2. Preliminaries. In this section, we introduce some notation and function spaces. All the results are known in $L^{2}$ norm (see [4]).

For $w \in D$, let $\varphi_{w}$ be the analytic map of $D$ onto $D$ defined by

$$
\varphi_{w}(z)=\frac{w-z}{1-\bar{w} z} .
$$

The Bergman metric on $D$ is defined by

$$
\beta(z, w)=\frac{1}{2} \ln \left(\frac{1+\left|\varphi_{w}(z)\right|}{1-\left|\varphi_{w}(z)\right|}\right) .
$$

For $r>0$ and $z \in D$, the Bergman disk centered at $z$ of radius $r$ is defined by

$$
E(z, r)=\{w \in D: \beta(w, z)<r\} .
$$

Let $|E(z, r)|=A(E(z, r))$. For fixed $r>0$, let

$$
\widehat{f}(z, r)=|E(z, r)|^{-1} \int_{E(z, r)} f(w) d A(w)
$$

and

$$
\mathrm{MO}_{p}(f, z, r)=|E(z, r)|^{-1} \int_{E(z, r)}|f(w)-\widehat{f}(z, r)|^{p} d A(w) .
$$

We say that $f$ is in $\mathrm{BMO}^{p, r}$ if $\mathrm{MO}_{p}(f, z, r)$ is a bounded function of $z$. We say that $f$ is in $\mathrm{VMO}_{\partial}^{p, r}$ if $\mathrm{MO}_{p}(f, z, r) \rightarrow 0$ as $|z| \rightarrow 1^{-}$. By Hölder's inequality, we have $\mathrm{MO}_{p}(f, z, r) \leq \mathrm{MO}_{q}(f, z, r)$ for $p \leq q$. Hence $\mathrm{BMO}^{q, r} \subset \mathrm{BMO}^{p, r}$ and $\mathrm{VMO}_{\partial}^{q, r} \subset \mathrm{VMO}_{\partial}^{p, r}$ for $p \leq q$. 
We will show that $\mathrm{BMO}^{p, r}$ and $\mathrm{VMO}_{\partial}^{p, r}$ actually do not depend on $r$. To achieve this goal, we need two more function spaces.

Let $\operatorname{OSC}(f, z)=\sup _{w \in E(z, 1)}|f(z)-f(w)|$ and let

$$
\begin{aligned}
\mathrm{BO} & =\{f \text { continuous on } D: \operatorname{OSC}(f, z) \text { is bounded on } D\}, \\
\mathrm{VO}_{\partial} & =\left\{f \text { continuous on } D: \operatorname{OSC}(f, z) \rightarrow 0 \text { as }|z| \rightarrow 1^{-}\right\} .
\end{aligned}
$$

By Corollary to Theorem 13 of [4], $E(z, 1)$ can be replaced by any $E(z, r)$ for $r>0$ in the definition above.

Finally, let

$$
\begin{aligned}
& \mathrm{F}^{p}=\left\{f \in L^{p}:|E(z, 1)|^{-1} \int_{E(z, 1)}|f(w)|^{p} d A(w) \text { is bounded on } D\right\}, \\
& \mathrm{F}_{\partial}^{p}=\left\{f \in L^{p}:|E(z, 1)|^{-1} \int_{E(z, 1)}|f(w)|^{p} d A(w) \rightarrow 0 \text { as }|z| \rightarrow 1^{-}\right\} .
\end{aligned}
$$

Hölder's inequality gives $\mathrm{F}^{q} \subset \mathrm{F}^{p}$ and $\mathrm{F}_{\partial}^{q} \subset \mathrm{F}_{\partial}^{p}$ for $p \leq q$. Note that $E(z, 1)$ can be replaced by any $E(z, r)$ in the definition above (see [6]).

We now introduce norms on these spaces. Let

$$
\begin{gathered}
\|f\|_{\mathrm{BMO}^{p}}=\sup _{z \in D}\left\{\operatorname{MO}_{p}(f, z, 1)\right\}^{1 / p}, \quad\|f\|_{\mathrm{BO}}=\sup _{z \in D} \operatorname{OSC}(f, z), \\
\|f\|_{\mathrm{F}^{p}}=\sup _{z \in D}\left\{|E(z, 1)|^{-1} \int_{E(z, 1)}|f(w)|^{p} d A(w)\right\}^{1 / p} .
\end{gathered}
$$

We use $c$ throughout the paper for any positive constants that may depend on the parameter $p$, but do not depend on $r$, where $r$ is the radius of the Bergman disk. If a positive constant depends on $r$, it will be denoted by $c(r)$.

The following lemma is analogous to Theorems 18 and 19 of [4].

Lemma 1. Let $1 \leq p<\infty$. Then

(i) $\mathrm{BMO}^{p, r}=\mathrm{BO}+\mathrm{F}^{p}$.

(ii) $\mathrm{VMO}_{\partial}^{p, r}=\mathrm{VO}_{\partial}+\mathrm{F}_{\partial}^{p}$.

Proof. To prove (i), we first prove that $\mathrm{BMO}^{p, r} \subset \mathrm{BO}+\mathrm{F}^{p}$. Suppose that $f \in \mathrm{BMO}^{p, r}$. Let $z \in D$ and $\widehat{f}(z)=\widehat{f}(z, r / 2)$. Then by Hölder's inequality,

$$
\begin{aligned}
& |\widehat{f}(z)-\widehat{f}(w)|^{p} \\
& \quad \leq|E(z, r / 2)|^{-1}|E(w, r / 2)|^{-1} \int_{E(z, r / 2)} \int_{E(w, r / 2)}|f(u)-f(w)|^{p} d A(u) d A(w),
\end{aligned}
$$

and for $\beta(w, z) \leq r / 2$, we have

$$
|\widehat{f}(z)-\widehat{f}(w)|^{p} \leq c(r)|E(z, r)|^{-2} \int_{E(z, r)} \int_{E(z, r)}|f(u)-f(w)|^{p} d A(u) d A(w) .
$$


Further,

$$
\begin{aligned}
& |E(z, r)|^{-2} \int_{E(z, r)} \int_{E(z, r)}|f(u)-f(w)|^{p} d A(u) d A(w) \\
& =|E(z, r)|^{-2} \int_{E(z, r)} \int_{E(z, r)}|f(u)-\widehat{f}(z, r)+\widehat{f}(z, r)-f(w)|^{p} d A(u) d A(w) \\
& \leq 2^{p-1}|E(z, r)|^{-2} \\
& \quad \times \int_{E(z, r)} \int_{E(z, r)}\left(|f(u)-\widehat{f}(z, r)|^{p}+|f(w)-\widehat{f}(z, r)|^{p}\right) d A(u) d A(w) \\
& =2^{p} \operatorname{MO}_{p}(f, z, r) .
\end{aligned}
$$

Therefore for $\beta(w, z) \leq r / 2$,

$$
|\widehat{f}(z)-\widehat{f}(w)| \leq 2^{p} c(r) \mathrm{MO}_{p}(f, z, r) .
$$

Thus $\widehat{f}$ is in BO.

Next we show that $f-\widehat{f}$ is in $\mathrm{F}^{p}$. Use Minkowski's inequality to get

$$
\begin{aligned}
\left\{|E(z, r / 2)|^{-1}\right. & \left.\int_{E(z, r / 2)}|f(w)-\widehat{f}(w)|^{p} d A(w)\right\}^{1 / p} \\
\leq & \left\{|E(z, r / 2)|^{-1} \int_{E(z, r / 2)}|f(w)-\widehat{f}(z)|^{p} d A(w)\right\}^{1 / p} \\
& +\left\{|E(z, r / 2)|^{-1} \int_{E(z, r / 2)}|\widehat{f}(z)-\widehat{f}(w)|^{p} d A(w)\right\}^{1 / p} .
\end{aligned}
$$

It is easy to check that both terms above on the right are dominated by $\mathrm{MO}_{p}(f, z, r)$. So $f-\widehat{f} \in \mathrm{F}^{p}$. This shows that $\mathrm{BMO}^{p, r} \subset \mathrm{BO}+\mathrm{F}^{p}$.

Now we show that $\mathrm{BO}+\mathrm{F}^{p} \subset \mathrm{BMO}^{p, r}$. By

$$
\mathrm{MO}_{p}(f, z, r) \leq|E(z, r)|^{-2} \int_{E(z, r)} \int_{E(z, r)}|f(w)-f(u)|^{p} d A(w) d A(u),
$$

we conclude that $\mathrm{BO} \subset \mathrm{BMO}^{p, r}$. From

$$
\mathrm{MO}_{p}(f, z, r) \leq 2^{p}|E(z, r)|^{-1} \int_{E(z, r)}|f(w)|^{p} d A(w),
$$

we conclude that $\mathrm{F}^{p} \subset \mathrm{BMO}^{p, r}$. Hence $\mathrm{BO}+\mathrm{F}^{p} \subset \mathrm{BMO}^{p, r}$. This proves (i).

The argument above implies (ii) if we take the limit as $|z| \rightarrow 1^{-}$.

Remarks. (i) Lemma 1 shows that $\mathrm{BMO}^{p, r}$ and $\mathrm{BMO}_{\partial}^{p, r}$ are independent of $r$ and hence will be denoted by $\mathrm{BMO}^{p}$ and $\mathrm{VMO}_{\partial}^{p}$, respectively.

(ii) For $f \in \mathrm{BMO}^{p}$ (or $\mathrm{VMO}^{p}$ ), a typical decomposition of $f$ is $f=$ $\widehat{f}+f-\widehat{f}$, where $\widehat{f} \in \mathrm{BO}$ (or $\mathrm{VO}_{\partial}$ ) and $f-\widehat{f} \in \mathrm{F}^{p}$ (or $\mathrm{F}_{\partial}$ ). 
(iii) If $p \geq 2$, then $\mathrm{BMO}^{p} \subset \mathrm{BMO}^{2}$ and $\mathrm{VMO}^{p} \subset \mathrm{VMO}^{2}$. Let

$$
\widetilde{f}(z)=\left(1-|z|^{2}\right)^{2} \int_{D} f(w)|1-z \bar{w}|^{4} d A(w)
$$

be the Berezin transform of $f$. Then Theorem 20 of [4] shows that we can also decompose $f \in \mathrm{BMO}^{p}$ (or $\mathrm{VMO}^{p}$ ) for $p \geq 2$ as $f=\widetilde{f}+f-\widetilde{f}$ such that $\tilde{f} \in \mathrm{BO}\left(\right.$ or $\left.\mathrm{VO}_{\partial}\right), f-\tilde{f} \in \mathrm{F}^{p}$ (or $\mathrm{F}_{\partial}$ ) and

$$
\|\tilde{f}\|_{\mathrm{BO}} \leq c\|f\|_{\mathrm{BMO}^{p}}, \quad\|f-\tilde{f}\|_{\mathrm{F}^{p}} \leq c\|f\|_{\mathrm{BMO}^{p}} .
$$

We need two more lemmas. The proof of the first lemma is similar to one given in [2] (see pages 5 and 6 ) and will be omitted.

Lemma 2. Let $1<p<\infty$ and $t(w)=\left(1-|w|^{2}\right)^{-1 / p p^{\prime}}$, where $w \in D$ and $1 / p+1 / p^{\prime}=1$. Then

$$
\begin{aligned}
& \int_{D} t(w)^{p^{\prime}} \beta(z, w)|1-z \bar{w}|^{-2} d A(w) \leq c t(z)^{p^{\prime}}, \\
& \int_{D} t(w)^{p} \beta(z, w)|1-z \bar{w}|^{-2} d A(w) \leq c t(z)^{p} .
\end{aligned}
$$

The other lemma is a special case of Theorem 2.2 of [6].

Lemma 3. Let $1 \leq p<\infty$ and $\alpha>-1$. If $f \in \mathrm{F}^{p}$, then

$$
\int_{D}|g(z)|^{p}\left(1-|z|^{2}\right)^{\alpha}|f(z)|^{p} d A(z) \leq c\|f\|_{\mathrm{F}^{p}}^{p} \int_{D}|g(z)|^{p}\left(1-|z|^{2}\right)^{\alpha} d A(z)
$$

for all analytic functions $g$ on $D$.

3. An upper bound condition. In this section, we give a sufficient condition for $C_{f}=M_{f} P-P M_{f}$, where

$$
P(g)(z)=\int_{D} g(w) K(z, w) d A(w),
$$

to be bounded or compact on $L^{p}$. The following upper bound condition for the kernel function $K$ is needed for the sufficiency:

$$
|K(z, w)| \leq c|1-z \bar{w}|^{-2}, \quad z, w \in D .
$$

We have the following theorem.

TheOrem 1. Let $2 \leq p<\infty$ and $K$ satisfy (4).

(i) If $f \in \mathrm{BMO}^{p}$, then $C_{f}$ is bounded on $L^{p}$.

(ii) If $f \in \mathrm{VMO}_{\partial}^{p}$, then $C_{f}$ is compact on $L^{p}$.

Proof. First let us prove (i). Suppose that $f \in \mathrm{BMO}^{p}$ for $2 \leq p<\infty$. Then by Remark (iii) following Lemma $1, f=\widetilde{f}+h$, where $h=f-\widetilde{f} \in$ $\mathrm{F}^{p}$. We will show that both $C_{\widetilde{f}}$ and $C_{h}$ are bounded on $L^{p}$. Note that $C_{\widetilde{f}}$ 
is an integral operator with the kernel function $(\widetilde{f}(z)-\widetilde{f}(w)) K(z, w)$. By Corollary 1 of [4], for $z, w \in D$ we have

$$
|(\widetilde{f}(z)-\widetilde{f}(w)) K(z, w)| \leq c\|f\|_{\mathrm{BMO}} \beta(z, w)|1-z \bar{w}|^{-2} .
$$

In view of Lemma 2 and the Schur test, $C_{\widetilde{f}}$ is bounded on $L^{p}$. Moreover, $\left\|C_{\widetilde{f}}\right\| \leq c\|f\|_{\mathrm{BMO}^{p}}$.

To show that $C_{h}$ is bounded, note that

$$
C_{h}=M_{h} P-P M_{h}=M_{h} P-\left(M_{\bar{h}} P^{*}\right)^{*},
$$

where $P^{*}$ is an integral operator with the kernel function $\overline{K(w, z)}$ on $L^{p^{\prime}}$ where $1 / p+1 / p^{\prime}=1$. For $g \in L^{p}$, we have

$$
\left|M_{h} P(g)(z)\right|^{p} \leq c\left|h(z) \int_{D}\right| g(w)|\cdot| 1-\left.\left.z \bar{w}\right|^{-2} d A(w)\right|^{p} .
$$

Apply Hölder's inequality to the right side above to get

$$
\begin{aligned}
\left|M_{h} P(g)(z)\right|^{p} \leq & c|h(z)|^{p}\left(\int_{D}|g(w)|^{p} t(w)^{-p}|1-z \bar{w}|^{-2} d A(w)\right) \\
& \times\left(\int_{D} t(w)^{p^{\prime}}|1-z \bar{w}|^{-2} d A(w)\right)^{p-1} \\
\leq & c|h(z)|^{p} t(z)^{p} \int_{D}|g(w)|^{p} t(w)^{-p}|1-z \bar{w}|^{-2} d A(w),
\end{aligned}
$$

where $t(w)$ is defined as in Lemma 2. Thus we have

$$
\begin{aligned}
\int_{D}\left|M_{h} P(g)(z)\right|^{p} d A(z) & \\
& \leq c \int_{D}|g(w)|^{p}|t(w)|^{-p} d A(w) \cdot \int_{D}|h(z)|^{p} t(z)^{p}|1-z \bar{w}|^{-2} d A(z) \\
& \leq c \int_{D}|g(w)|^{p}|t(w)|^{-p} d A(w) \cdot\|h\|_{\mathrm{F}^{p}}^{p} \int_{D} t(z)^{p}|1-z \bar{w}|^{-2} d A(z) \\
& \leq c\|f\|_{\mathrm{BMO}^{p}}^{p} \int_{D}|g(w)|^{p} d A(w),
\end{aligned}
$$

where we used Lemma 3 in the second step above and (3) in the last step. Therefore $M_{h} P$ is bounded on $L^{p}$. Since $p \geq 2, p \geq p^{\prime}$. So $\bar{h} \in \mathrm{F}^{p^{\prime}}$. The same argument yields that $\left(M_{\bar{h}} P^{*}\right)$ is bounded on $L^{p^{\prime}}$, therefore $\left(M_{\bar{h}} P^{*}\right)^{*}$ is bounded on $L^{p}$. This proves that $C_{f}=C_{\widetilde{f}}+C_{h}$ is bounded on $L^{p}$ and gives $\left\|C_{f}\right\| \leq c\|f\|_{\mathrm{BMO}^{p}}$.

Now we prove (ii). Suppose that $f \in \mathrm{VMO}^{p}$ for $2 \leq p<\infty$. Let $f=\widetilde{f}+h$, where $h=f-\widetilde{f} \in \mathrm{F}_{\partial}$. We show that both $C_{\widetilde{f}}$ and $C_{h}$ are compact on $L^{p}$. 
For $0<\delta<1$, let $C_{\widetilde{f}, \delta}$ be the integral operator with kernel function

$$
K_{\widetilde{f}, \delta}(z, w)=(\widetilde{f}(z)-\widetilde{f}(w)) \chi_{\{|z|<\delta\}}(z, w) K(z, w) .
$$

If $|z|<\delta<1$ and $w \in D$, then $|K(z, w)| \leq c$ and hence

$$
\begin{aligned}
|(\widetilde{f}(z)-\widetilde{f}(w)) K(z, w)| & \leq c\|f\|_{\mathrm{BMO}} \beta(z, w) \leq c\|f\|_{\mathrm{BMO}}(\beta(z, 0)+\beta(w, 0)) \\
& \leq c\|f\|_{\mathrm{BMO}}(1+\ln (1 /(1-|w|)) .
\end{aligned}
$$

It is clear that

$$
\int_{D}\left(\int_{D}\left|K_{\tilde{f}, \delta}(z, w)\right|^{p} d A(z)\right)^{p^{\prime} / p} d A(w)<\infty .
$$

Thus for every $\delta<1, C_{\widetilde{f}, \delta}$ is a compact operator on $L^{p}$ by Ex. 7 in Section 6.3 of [5]. Given $\varepsilon>0$, by the proof of Lemma 26 of [4], there is $\delta \in(0,1)$ such that for $\delta<|z|<1$,

$$
|(\widetilde{f}(z)-\widetilde{f}(w)) K(z, w)| \leq c \varepsilon\|f\|_{\mathrm{BMO}} \beta(z, w)|1-z \bar{w}|^{-2} .
$$

Therefore $\left\|C_{\widetilde{f}}-C_{\widetilde{f}, \delta}\right\| \leq c \varepsilon\|f\|_{\text {BMO }}$ by the proof in part (i) and hence $\left\|C_{\widetilde{f}}-C_{\widetilde{f}, \delta}\right\| \rightarrow 0$ as $\delta \rightarrow 1^{-}$. This shows that $C_{\widetilde{f}}$ is compact on $L^{p}$.

To prove that $C_{h}$ is compact, let $h_{\delta}(z)=h(z) \chi_{\{|z|<\delta\}}(z)$ for $0<\delta<1$. Then $M_{h_{\delta}} P$ is an integral operator with the kernel $h_{\delta}(z) K(z, w)$. Since $\left|h_{\delta}(z) K(z, w)\right| \leq c\left|h_{\delta}(z)\right|$ for $z, w \in D$, it is clear that $M_{h_{\delta}} P$ is a compact operator on $L^{p}$. By the argument in part (i) that is used to prove that $M_{h} P$ is bounded, for $g \in L^{p}$ we have

$$
\int_{D}\left|M_{h-h_{\delta}} P(g)(z)\right|^{p} d A(z) \leq c \sup _{|z| \geq \delta}\left(|E(z, 1)|^{-1} \int_{E(z, 1)}|h(w)|^{p} d A(w)\right)\|g\|_{p}^{p} .
$$

Thus $\left\|M_{h} P-M_{h_{\delta}} P\right\|=\left\|M_{h-h_{\delta}} P\right\| \rightarrow 0$ as $\delta \rightarrow 1^{-}$, since $h \in \mathrm{F}_{\partial}^{p}$. So $M_{h} P$ is compact. The same argument implies that $\left(M_{\bar{h}} P^{*}\right)$ is compact on $L^{p^{\prime}}$, since $\bar{h} \in \mathrm{F}_{\partial}^{p^{\prime}}$. Hence $P M_{h}=\left(M_{\bar{h}} P^{*}\right)^{*}$ is compact on $L^{p}$. This shows that $C_{h}=M_{h} P-P M_{h}$ is compact on $L^{p}$ and completes the proof.

4. A local bound condition. In this section, we prove the converse of Theorem 1 if the kernel function satisfies the following local bound condition:

$$
K^{-1}(u, w)=K^{-1}(z, z)+R(u, w, z), \quad u, w \in E(z, r), z \in D,
$$

such that

$$
K^{-1}(z, z) \approx\left(1-|z|^{2}\right)^{2}, \quad|R(u, w, z)| \leq \operatorname{cr}\left(1-|z|^{2}\right)^{2}, \quad u, w \in E(z, r),
$$

where $r$ is considered to be small enough and $K^{-1}(z, z) \approx\left(1-|z|^{2}\right)^{2}$ means that

$$
0<c_{1} \leq \frac{K^{-1}(z, z)}{\left(1-|z|^{2}\right)^{2}} \leq c_{2}
$$


We have the following theorem.

TheOREM 2. Let $1<p<\infty$ and $K$ satisfy (5) for all $r$ small enough.

(i) If $C_{f}$ is bounded on $L^{p}$, then $f \in \mathrm{BMO}^{p}$.

(ii) If $C_{f}$ is compact on $L^{p}$, then $f \in \mathrm{VBO}_{\partial}^{p}$.

Proof. For $z \in D$, we have

$$
\begin{aligned}
& \mathrm{MO}_{p}(f, z, r) \\
& =|E(z, r)|^{-1-p} \int_{E(z, r)}\left|\int_{E(z, r)}(f(u)-f(w)) K(u, w) K^{-1}(u, w) d A(w)\right|^{p} d A(u) .
\end{aligned}
$$

By (5) we have

$$
\begin{aligned}
\operatorname{MO}_{p}(f, z, r) & \\
\leq & 2^{p-1}|E(z, r)|^{-1-p} \\
& \times \int_{D}\left|\int_{E(z, r)}(f(u)-f(w)) K(u, w) K^{-1}(z, z) d A(w)\right|^{p} d A(u) \\
& +2^{p-1}|E(z, r)|^{-1-p} \\
& \times \int_{E(z, r)}\left|\int_{E(z, r)}(f(u)-f(w)) K(u, w) R(u, w, z) d A(w)\right|^{p} d A(u) \\
\leq & c(r)\left(1-|z|^{2}\right)^{-2}\left\|C_{f}\left(\chi_{E(z, r)}\right)\right\|_{p}^{p} \\
& +2^{p-1}|E(z, r)|^{-1-p} \\
& \times \int_{E(z, r)}\left(\int_{E(z, r)}|f(u)-f(w)| \cdot|K(u, w) R(u, w, z)| d A(w)\right)^{p} d A(u),
\end{aligned}
$$

where we used the fact that $|E(z, r)| \approx r^{2}\left(1-|z|^{2}\right)^{2}$ in the last step. If we denote the second term above by $T(f, p, z, r)$, then

$$
\mathrm{MO}_{p}(f, z, r) \leq c(r)\left(1-|z|^{2}\right)^{-2}\left\|C_{f}\left(\chi_{E(z, r)}\right)\right\|_{p}^{p}+T(f, p, z, r) .
$$

By (5), for $u, w \in E(z, r)$ and $r$ small enough,

$$
\begin{aligned}
|K(u, w)| & =\left|\left(K^{-1}(z, z)+R(u, w, z)\right)^{-1}\right| \\
& \leq\left(K^{-1}(z, z)-|R(u, w, z)|\right)^{-1} \leq c\left(1-|z|^{2}\right)^{-2} .
\end{aligned}
$$

Therefore we have, for $r$ small enough,

$$
|K(u, w) R(u, w, z)| \leq c r, \quad u, w \in E(z, r) .
$$

By the estimate above and Hölder's inequality, for $r$ small enough we have 


$$
\begin{aligned}
& T(f, p, z, r) \\
& \leq c r^{p}|E(z, r)|^{-1-p} \int_{E(z, r)}\left(\int_{E(z, r)}|f(u)-f(w)| d A(w)\right)^{p} d A(u) \\
& \leq c r^{p}|E(z, r)|^{-2} \int_{E(z, r)} \int_{E(z, r)}|f(u)-f(w)|^{p} d A(w) d A(u) \\
& \leq c r^{p}|E(z, r)|^{-2} \\
& \quad \times \int_{E(z, r) E(z, r)} 2^{p-1}\left(|f(u)-\widehat{f}(z, r)|^{p} e+|f(w)-\widehat{f}(z, r)|^{p}\right) d A(w) d A(u) \\
& =2^{p} c r^{p} \operatorname{MO}_{p}(f, z, r) .
\end{aligned}
$$

Now we choose $r$ small enough such that $1-2^{p} c r^{p}>1 / 2$ where $c$ is the constant given in the last step. Then by (6) we have

$$
\begin{aligned}
\frac{1}{2} \mathrm{MO}_{p}(f, z, r) & \leq\left(1-2^{p} c r^{p}\right) \mathrm{MO}_{p}(f, z, r) \\
& \leq c(r)\left(1-|z|^{2}\right)^{-2}\left\|C_{f}\left(\chi_{E(z, r)}\right)\right\|_{p}^{p} \\
& =c(r)\left\|C_{f}\left(\left(1-|z|^{2}\right)^{-2 / p} \chi_{E(z, r)}\right)\right\|_{p}^{p} .
\end{aligned}
$$

Suppose that $C_{f}$ is bounded. Since $\left(1-|z|^{2}\right)^{-2 / p} \chi_{E(z, r)}$ is $L^{p}$-norm bounded, $\left\|C_{f}\left(\left(1-|z|^{2}\right)^{-2 / p} \chi_{E(z, r)}\right)\right\|_{p}^{p}$ is bounded for $z \in D$. This shows that $f \in \mathrm{BMO}^{p}$.

Suppose that $C_{f}$ is compact. Since $\left(1-|z|^{2}\right)^{-2 / p} \chi_{E(z, r)}$ is $L^{p}$-norm bounded and tends to zero uniformly on compact subsets of $D$ as $|z| \rightarrow 1^{-}$, $\left(1-|z|^{2}\right)^{-2 / p} \chi_{E(z, r)}$ tends to zero weakly in $L^{p}$ as $|z| \rightarrow 1^{-}$. Thus

$$
\left\|C_{f}\left(\left(1-|z|^{2}\right)^{-2 / p} \chi_{E(z, r)}\right)\right\|_{p}^{p} \rightarrow 0
$$

as $|z| \rightarrow 1^{-}$. This shows that $f \in \mathrm{VMO}_{\partial}^{p}$ and the proof is now complete.

5. Examples. In this section, we give some kernel functions that satisfy both (4) and (5).

(a) Analytic Bergman kernel:

$$
K_{\mathrm{a}}(z, w)=\frac{1}{(1-z \bar{w})^{2}} .
$$

Clearly, $K_{\mathrm{a}}$ satisfies (4). Note that $K_{\mathrm{a}}^{-1}(z, z)=\left(1-|z|^{2}\right)^{2}$ for $z \in D$. Let $R(u, w, z)=(1-u \bar{w})^{2}-\left(1-|z|^{2}\right)^{2}$. Thus

$$
R(u, w, z)=\left(|z|^{2}-u \bar{w}\right)\left((1-u \bar{w})+\left(1-|z|^{2}\right)\right) .
$$

It is an easy computation that if $u \in E(z, r)$ and $r \leq 1 / 2$, then

$$
|u-z|<\operatorname{cr}\left(1-|z|^{2}\right) \text {. }
$$


So for $u, w \in E(z, r)$ and $r \leq 1 / 2$,

$$
\left.|| z\right|^{2}-u \bar{w}|=|-\bar{z}(u-z)-u(\overline{w-z}) \mid \leq \operatorname{cr}\left(1-|z|^{2}\right) .
$$

By (7) we have

$$
|1-u \bar{w}|=\left|\left(1-|z|^{2}\right)+\left(|z|^{2}-u \bar{w}\right)\right| \leq c\left(1-|z|^{2}\right) .
$$

Therefore for $u, w \in E(z, r)$ and $r \leq 1 / 2$,

$$
|R(u, w, z)|=\left.|| z\right|^{2}-u \bar{w}|\cdot|(1-u \bar{w})+\left(1-|z|^{2}\right) \mid \leq c r\left(1-|z|^{2}\right)^{2} .
$$

This shows that $K_{\mathrm{a}}$ satisfies (5).

Let $C_{f}^{\mathrm{a}}=M_{f} P-P M_{f}$, where $P$ is an integral operator with the kernel function $K_{\mathrm{a}}$.

Theorems 1 and 2 give the following known result.

Corollary 1. Let $2 \leq p<\infty$. Then

(i) $C_{f}^{\text {a }}$ is bounded on $L^{p} \Leftrightarrow f \in \mathrm{BMO}^{p}$;

(ii) $C_{f}^{\mathrm{a}}$ is compact on $L^{p} \Leftrightarrow f \in \mathrm{VMO}_{\partial}^{p}$.

Let $H_{f}^{\mathrm{a}}=(I-P) M_{f} P$ be the Hankel operator. We have the following relations between $C_{f}^{\mathrm{a}}$ and $H_{f}^{\mathrm{a}}$ :

$$
C_{f}^{\mathrm{a}}=H_{f}^{\mathrm{a}}-\left(H_{\bar{f}}^{\mathrm{a}}\right)^{*}, \quad H_{f}^{\mathrm{a}}=(I-P) C_{f}^{\mathrm{a}}, \quad\left(H_{f}^{\mathrm{a}}\right)^{*}=-C_{f}^{\mathrm{a}}(I-P) .
$$

Thus Corollary 1 is equivalent to the following result.

Corollary 2. Let $2 \leq p<\infty$ and $1 / p+1 / p^{\prime}=1$. Then

(i) $H_{f}^{\mathrm{a}}$ is bounded on $L^{p}$ and $H_{\bar{f}}^{\mathrm{a}}$ is bounded on $L^{p^{\prime}} \Leftrightarrow f \in \mathrm{BMO}^{p}$.

(ii) $H_{f}^{\mathrm{a}}$ is compact on $L^{p}$ and $H_{\bar{f}}^{\mathrm{a}}$ is compact on $L^{p^{\prime}} \Leftrightarrow f \in \mathrm{VMO}_{\partial}^{p}$.

(b) Harmonic Bergman kernel:

$$
K_{\mathrm{h}}(z, w)=2 \Re \frac{1}{(1-z \bar{w})^{2}}-1 .
$$

Clearly, $K_{\mathrm{h}}$ satisfies (4). In order to show that $K_{\mathrm{h}}$ satisfies (5), we need the following proposition.

Proposition. For $r$ small enough, we have

$$
K_{\mathrm{h}}^{-1}(u, w)=K_{\mathrm{h}}^{-1}(z, z)+R(u, w, z), \quad u, w \in E(z, r), z \in D,
$$

where

$$
|R(u, w, z)| \leq \operatorname{cr}\left(1-|z|^{2}\right)^{2}, \quad u, w \in E(z, r) .
$$

Proof. Using the formula for $K_{\mathrm{h}}(z, w)$ given on page 427 of [8], we have

$$
K_{\mathrm{h}}^{-1}(u, w)=\frac{|1-u \bar{w}|^{4}}{\left(1-|u|^{2}|w|^{2}\right)^{2}-2|u|^{2}|w|^{2}|1-u \bar{w}|^{2}} \equiv \frac{A(u, w)}{B(u, w)} .
$$


Since

$$
K_{\mathrm{h}}^{-1}(z, z)=\frac{\left(1-|z|^{2}\right)^{2}}{2-\left(1-|z|^{2}\right)^{2}},
$$

it is easy to show that

$$
(1 / 2)\left(1-|z|^{2}\right)^{2} \leq K_{\mathrm{h}}^{-1}(z, z) \leq\left(1-|z|^{2}\right)^{2} .
$$

Let $u=x_{1}+i x_{2}, w=x_{3}+i x_{4}$. We will expand $K_{\mathrm{h}}^{-1}(u, w)$ at the point $(z, z)$ for $z \in D$. For $u, w \in E(z, r)$, since $R(u, w, z)=K_{\mathrm{h}}^{-1}(u, w)-$ $K_{\mathrm{h}}^{-1}(z, z)$, we have

$$
\begin{aligned}
|R(u, w, z)| \leq & \sum_{i=1}^{2} \sup _{u, w \in E(z, r)}\left|\left(K_{\mathrm{h}}^{-1}\right)_{x_{i}}(u, w)\right| \cdot|u-z| \\
& +\sum_{i=3}^{4} \sup _{u, w \in E(z, r)}\left|\left(K_{\mathrm{h}}^{-1}\right)_{x_{i}}(u, w)\right| \cdot|w-z| .
\end{aligned}
$$

For $i=1,2,3,4$,

$$
\left(K_{\mathrm{h}}^{-1}\right)_{x_{i}}(u, w)=\frac{A_{x_{i}}(u, w) B(u, w)-A(u, w) B_{x_{i}}(u, w)}{B^{2}(u, w)} .
$$

It is straightforward to check that for $u, w \in E(z, r), r<1 / 2$, and $i=$ $1,2,3,4$,

$$
\begin{aligned}
\left|A_{x_{i}}(u, w)\right| & \leq c\left(1-|z|^{2}\right)^{3}, & \left|B_{x_{i}}(u, w)\right| & \leq c\left(1-|z|^{2}\right), \\
|A(u, w)| & \leq c\left(1-|z|^{2}\right)^{4}, & |B(u, w)| & \leq c\left(1-|z|^{2}\right)^{2},
\end{aligned}
$$

therefore

$$
\left|A_{x_{i}}(u, w) B(u, w)-A(u, w) B_{x_{i}}(u, w)\right| \leq c\left(1-|z|^{2}\right)^{5} .
$$

Next we will obtain a lower bound for $B(u, w)$ for $u, w \in E(z, r)$. We have

$$
\begin{aligned}
B(u, w) & \\
= & \left(1-|u|^{2}|w|^{2}\right)^{2}-2|u|^{2}|w|^{2}|1-u \bar{w}|^{2} \\
= & \left(1-|u|^{2}|w|^{2}\right)^{2}-2|u|^{2}|w|^{2}|(1-|u| \cdot|w|)+(|u| \cdot|w|-u \bar{w})|^{2} \\
\geq & \left(1-|u|^{2}|w|^{2}\right)^{2}-2|u|^{2}|w|^{2}((1-|u| \cdot|w|)+|| u|\cdot| w|-u \bar{w}|)^{2} \\
= & \underbrace{\left(1-|u|^{2}|w|^{2}\right)^{2}-2|u|^{2}|w|^{2}(1-|u| \cdot|w|)^{2}}_{C(u, w)} \\
& -(\underbrace{4|u|^{2}|w|^{2}(1-|u| \cdot|w|)|| u|\cdot| w|-u \bar{w}|+2|u|^{2}|w|^{2}|| u|\cdot| w|-u \bar{w}|^{2}}_{D(u, w)}) \\
= & C(u, w)-D(u, w) .
\end{aligned}
$$


For $u, w \in E(z, r)$ and $r \leq 1 / 2,|u-z|<\operatorname{cr}\left(1-|z|^{2}\right),|w-z|<\operatorname{cr}\left(1-|z|^{2}\right)$, therefore for $r$ small enough, $1-|u|>c\left(1-|z|^{2}\right), 1-|w|>c\left(1-|z|^{2}\right)$. We have

$$
\begin{aligned}
C(u, w) & =(1-|u| \cdot|w|)^{2}\left(1+2|u| \cdot|w|-|u|^{2}|w|^{2}\right) \\
& \geq(1-|u| \cdot|w|)^{2} \geq(1-|u|)^{2} \geq c\left(1-|z|^{2}\right)^{2}
\end{aligned}
$$

for $u, w \in E(z, r)$ and $r$ small enough. By (7), for $u, w \in E(z, r)$ and $r \leq 1 / 2$ we get

$$
\left.|| z\right|^{2}-|u| \cdot|w||\leq||z|^{2}-u \bar{w} \mid \leq \operatorname{cr}\left(1-|z|^{2}\right),
$$

therefore

$$
|| u|\cdot| w|-u \bar{w}| \leq \operatorname{cr}\left(1-|z|^{2}\right) .
$$

Thus

$$
D(u, w) \leq 4(1-|u| \cdot|w|)|| u|\cdot| w|-u \bar{w}|+2|| u|\cdot| w|-u \bar{w}|^{2} \leq c r\left(1-|z|^{2}\right)^{2}
$$

for $u, w \in E(z, r)$ and $r \leq 1 / 2$. Therefore for $u, w \in E(z, r)$ and $r$ small enough,

$$
B(u, w) \geq c\left(1-|z|^{2}\right)^{2}-c r\left(1-|z|^{2}\right)^{2} \geq c\left(1-|z|^{2}\right)^{2} .
$$

Thus for $r$ small enough,

$$
\sup _{u, w \in E(z, r)}\left|\left(K_{\mathrm{h}}^{-1}\right)_{x_{i}}(u, w)\right| \leq c\left(1-|z|^{2}\right), \quad i=1,2,3,4 .
$$

Hence the desired estimate for $R(u, w, z)$ follows from (8).

Therefore $K_{\mathrm{h}}$ satisfies both (4) and (5). Let

$$
C_{f}^{\mathrm{h}}=M_{f} P-P M_{f}, \quad H_{f}^{\mathrm{h}}=(I-P) M_{f} P,
$$

where $P$ is an integral operator with the kernel function $K_{\mathrm{h}}$.

Theorems 1 and 2 imply the following result.

Corollary 3. Let $p \geq 2$. Then

(i) $C_{f}^{\mathrm{h}}$ is bounded on $L^{p} \Leftrightarrow f \in \mathrm{BMO}^{p}$.

(ii) $C_{f}^{\mathrm{h}}$ is compact on $L^{p} \Leftrightarrow f \in \mathrm{VMO}_{\partial}^{p}$.

Since $K_{\mathrm{h}}$ is real-valued, $H_{f}^{\mathrm{h}}$ is bounded or compact on $L^{p}$ iff $H_{\bar{f}}^{\mathrm{h}}$ is bounded or compact on $L^{p}$. Corollary 3 can now be rewritten as follows:

Corollary 4. Let $p \geq 2$ and $1 / p+1 / p^{\prime}=1$. Then

(i) $H_{f}^{\mathrm{h}}$ is bounded on $L^{p}$ and $H_{f}^{\mathrm{h}}$ is bounded on $L^{p^{\prime}} \Leftrightarrow f \in \mathrm{BMO}^{p}$.

(ii) $H_{f}^{\mathrm{h}}$ is compact on $L^{p}$ and $H_{f}^{\mathrm{h}}$ is compact on $L^{p^{\prime}} \Leftrightarrow f \in \mathrm{VMO}_{\partial}^{p}$.

Corollaries 3 and 4 generalize Theorems 2.1 and 2.3 of [9]. The author was informed that these generalizations have also been obtained by Zhijian Wu using a different method. 
(c) A nonorthogonal projection kernel:

$$
K_{\mathrm{n}}(z, w)=3 \frac{\left(1-|w|^{2}\right)^{2}}{(1-z \bar{w})^{4}} .
$$

See Lemma 1.17 of [2]. Clearly, $K_{\mathrm{n}}$ satisfies (4). We can use the same argument as for $K_{\mathrm{h}}(z, w)$ to show that $K_{\mathrm{n}}$ also satisfies (5). The details are omitted.

6. A related result. In this section, we consider the following integral operator:

$$
\bar{C}_{f}(g)(z)=\int_{D}|f(z)-f(w)| g(w) K(z, w) d A(w) .
$$

Let $r>0$ be fixed and $K$ satisfy the following local bound condition:

$$
K^{-1}(u, w) \approx\left(1-|z|^{2}\right)^{2}, \quad u, w \in E(z, r), z \in D .
$$

It is clear that condition (5) implies (9) if $K$ is real-valued.

The following is a similar result on $\bar{C}_{f}$.

Theorem 3. Let $2 \leq p<\infty$ and $K$ satisfy both (4) and (9). Then

(i) $\bar{C}_{f}$ is bounded on $L^{p} \Leftrightarrow f \in \mathrm{BMO}^{p}$.

(ii) $\bar{C}_{f}$ is compact on $L^{p} \Leftrightarrow f \in \mathrm{VMO}_{\partial}^{p}$.

Proof. " $\Leftarrow$ " This part of the proof is entirely the same as for Theorem 1 .

" $\Rightarrow$ " This part of the proof is significantly easier than that for Theorem 2 . We only prove (i) since the proof for (ii) is similiar. Suppose that $\bar{C}_{f}$ is bounded on $L^{p}$. Then for $z \in D$, we have

$$
\begin{aligned}
\mathrm{MO}_{p}(f, z, r)= & |E(z, r)|^{-1-p} \\
& \times \int_{E(z, r)}\left|\int_{E(z, r)}(f(u)-f(w)) K(u, w) K^{-1}(u, w) d A(w)\right|^{p} d A(u) \\
\leq & c\left(1-|z|^{2}\right)^{2 p}|E(z, r)|^{-1-p} \\
& \times \int_{E(z, r)}\left(\int_{E(z, r)}|f(u)-f(w)| K(u, w) d A(w)\right)^{p} d A(u) \\
\leq & c(r)\left\|\bar{C}_{f}\left(\left(1-|z|^{2}\right)^{-2 / p} \chi_{E(z, r)}\right)\right\|_{p}^{p},
\end{aligned}
$$

where we used $(9)$ in the second step and the fact $|E(z, r)| \approx\left(1-|z|^{2}\right)^{2}$ in the last step. Since $\left(1-|z|^{2}\right)^{-2 / p} \chi_{E(z, r)}$ is norm bounded on $L^{p}, \mathrm{MO}_{p}(f, z, r)$ is bounded for $z \in D$, i.e., $f$ is in $\mathrm{BMO}^{p}$.

Acknowledgments. Part of the paper was written when I was a Ph.D. student under the guidance of Sheldon Axler. I am grateful to him for his help and encouragement. 


\section{References}

[1] S. Axler, The Bergman space, the Bloch space, and commutators of multiplication operators, Duke Math. J. 53 (1986), 315-332.

[2] - Bergman spaces and their operators, in: Surveys of Some Recent Results in Operator Theory, Vol. 1, J. B. Conway and B. B. Morrel (eds.), Pitman Res. Notes Math. Ser. 171, Longman Sci. Tech., 1988, 1-50.

[3] F. Beatrous and S. Li, On the boundedness and compactness of operators of Hankel type, J. Funct. Anal. 111 (1993), 350-379.

[4] D. Békollé, C. A. Berger, L. A. Coburn and K. H. Zhu, BMO and the Bergman metric on bounded symmetric domains, ibid. 93 (1990), 310-350.

[5] J. B. Conway, A Course in Functional Analysis, Springer, New York, 1985.

[6] D. H. Luecking, Forward and reverse Carleson inequalities for functions in Bergman spaces and their derivatives, Amer. J. Math. 107 (1985), 85-111.

[7] - Characterizations of certain classes of Hankel operators on the Bergman spaces of the unit disc, J. Funct. Anal. 110 (1992), 247-271.

[8] J. Miao, Toeplitz operators on harmonic Bergman spaces, Integral Equations Operator Theory 27 (1997), 426-438.

[9] Z. Wu, Operators on harmonic Bergman spaces, ibid. 24 (1996), 352-371.

Department of Computer Science and Mathematics

Arkansas State University

P.O. Box 70

State University, AR 72467, U.S.A.

E-mail:miao@csm.astate.edu 